TRANSACTIONS OF THE

AMERICAN MATHEMATICAL SOCIETY

Volume 353, Number 1, Pages 95-115

S 0002-9947(00)02585-X

Article electronically published on August 9, 2000

\title{
DEGREE OF STRATA OF SINGULAR CUBIC SURFACES
}

\author{
RAFAEL HERNÁNDEZ AND MARÍA J. VÁZQUEZ-GALLO
}

\begin{abstract}
We determine the degree of some strata of singular cubic surfaces in the projective space $\mathbf{P}^{3}$. These strata are subvarieties of the $\mathbf{P}^{19}$ parametrizing all cubic surfaces in $\mathbf{P}^{3}$. It is known what their dimension is and that they are irreducible. In 1986, D. F. Coray and I. Vainsencher computed the degree of the 4 strata consisting on cubic surfaces with a double line. To work out the case of isolated singularities we relate the problem with (stationary) multiple-point theory.
\end{abstract}

\section{IntRodUCTION}

The purpose of this work is to determine the degree of certain strata of singular cubic surfaces in $\mathbf{P}_{\mathbf{C}}^{3}$. In the nineteenth century L. Schläfli [26] and A. Cayley 6] classified the cubic surfaces in $\mathbf{P}^{3}$ according to the type of their singularities. We consider this classification with the point of view given by J. W. Bruce and C. T. C. Wall 5 100 years later, based on the modern theory of singularities [2, [3.

Let us suppose that the cubic surfaces are irreducible and have only isolated singularities. Fixed a singular point $P$ of a cubic surface $C$, there are 4 types of surfaces, according to the rank of a quadratic form associated to each one of them: surfaces with a conic node, a binode, a uninode and a triple point at $P$.

Inside each one of these classes, the different singularities presented by a surface $C$ are determined by the possible coincidences of the 6 lines passing through the fixed singular point $P$ and contained in $C$.

Besides of these, there are 4 strata of irreducible surfaces with non isolated singularities (that are, in fact, ruled surfaces with a double line) and 9 of reducible cubic surfaces.

The strata resulting from this classification is the closure of quasi-projective subvarieties of the $\mathbf{P}^{19}$ parametrizing all cubic surfaces in $\mathbf{P}^{3}$. Regarding geometric properties of strata, it is known what their dimension is [5] and that the strata are irreducible 4]. Our aim here is to determine their degree.

First of all, the degree of strata parametrizing reducible surfaces is easy to compute [30]. In the case of the 4 big strata given by the rank of a quadratic form, the degree can be obtained at least in two ways [30], 28]. On the other hand, in 1986, D. F. Coray and I. Vainsencher calculated the degree of the 4 strata of ruled cubic surfaces having a double line [8]. Their work motivates ours.

Received by the editors March 15, 1999.

2000 Mathematics Subject Classification. Primary 14N05, 14C17; Secondary 14C05, 14 C15.

Key words and phrases. Enumerative geometry, strata of singular cubic surfaces, stationary multiple-point theory. 
In this paper, we determine the degree of 7 of the 11 strata of cubic surfaces with a conic node, using a method that must work, essentially in the same way, with some strata of cubics with a binode or a uninode.

Our approach to the problem is to relate it with stationary multiple-point theory, whose main goal is the enumeration of $k$-tuples of points of a scheme $Z$ with the same image under a morphism of schemes $f: Z \rightarrow S$ and such that the points have collapsed in accordance with a given partition of $k$.

We construct first some suitable parameter spaces and then we consider the lines whose coincidences determine the strata in our case, as stationary multiple points of a morphism between two of these parameter spaces.

One of the first examples of multiple-point formulas related with our problem is that of De Jonquières (1866), allowing us to consider coincidences of points in the zero scheme of sections of a line bundle on a given curve. One century later, I. Vainsencher 29] (1981) proved a generalized De Jonquières' formula, valid for a general line bundle. Some years after, S. J. Colley [7] obtained more formulas for stationary multiple points using the iteration method by S. L. Kleiman [16], 17], [18], who had already given some multiple-point formulas for the non-stationary cases.

We cannot apply Vainsencher's results in order to compute the strata's degree because we are dealing with coincidences of points in the plane. On the other hand, in Colley's work there are no formulas for the cases we need (stationary 6 -tuple points).

We use then the more general multiple-point formula by Z. Ran [24] (1984), based on the residual intersection formulas of W. Fulton and D. Laksov [10, that allows us to treat coincidences of points in the zero scheme, let's say $X$, of sections of a vector bundle with rank equal to $\operatorname{codim}(X)$. This turns out to be our case with a rank 2 vector bundle while in [24] all the examples worked out deal with line bundles.

Then, in $\$ 5$ we prove results justifying the computation of the degree of strata of cubic surfaces with a conic node, as a product of cycles in the intersection ring of a suitable space of 6 -tuples.

The cases not covered here are the strata given by a collapse with less than 3 different lines. Those correspond in our construction to 6-tuples of points that are not curvilinear (i.e. that don't live in a smooth curve) and, in general, the parameter space $Z_{k}$ used to enumerate them only parametrizes ordered and curvilinear $k$ tuples of a scheme $Z$ over another one $S$ [17, 24].

Regarding to the effective computation of these degrees, the method employed here leads to calculate in $A^{*}\left(Z_{k}\right)$ (the intersection ring of $Z_{k}$ ) the degree of a zerodimensional cycle, obtained by taking up cycles in $A^{*}\left(Z_{l}\right)$, with $l<k$, through the inductive construction of $Z_{k}$.

Given a morphism from $Z$ to $S$, if $A^{*}(S)$ is known and $A^{*}(Z)$ is a finitely generated $A^{*}(S)$-module, we are able to give an inductive method to compute the degree of zero-dimensional cycles of $A^{*}\left(Z_{k}\right)$ in the previous conditions (see Proposition 6.1).

We note that, whenever $Z$ is a projective bundle over $S$, we can explicitly describe the intersection ring of the space $Z_{k}$ (see $\$(6)$. In our case $A^{*}\left(Z_{6}\right)$ is a polynomial ring over $Z$ with 23 generators modulo 38 relations and effective computations in this ring, using Gröbner basis' method, is much more costly that our inductive method. 
We implement this method in a computer program written in Maple [20]. This program could be useful each time that one uses the parameter spaces $Z_{k}$ in order to study enumerative questions depending on what is happenning on the fibers of $Z$ over $S$.

This paper is organized as follows: $₫ 2$ is devoted to the classification of singular cubic surfaces in $\mathbf{P}^{3}$. In $\$$ we construct some parameter spaces appropriated for the problem. We deal in $\$$ with the spaces of $k$-tuples and the secant bundles of Ran's work 24]. In \$5 we state and prove our main result giving the degree of some strata of cubic surfaces with a conic node. Finally, in 6 we work with the intersection ring of spaces of $k$-tuples and the effective computation of strata's degree.

This work is part of the second author's Ph.D. Thesis. We would like to thank E. Arrondo and I. Vainsencher for suggestions and comments.

Notation 1.1. We shall tacitly assume that all schemes are defined over an algebraically closed field of characteristic 0 . Our notation follows the book of W. Fulton [9], in particular we will write $P(E)$ for the projectivization of a vector bundle $E$ over a scheme $Z$, defined as $P(E)=\operatorname{Proj}\left(\operatorname{Sym}^{*}\right)$. If $Z$ is a non-singular scheme, the intersection ring we shall use is the Chow ring of classes of rational equivalence of cycles, graduated by codimension and denoted by $A^{*}(Z)$. We will denote by $G r$ the grassmannian of lines of $\mathbf{P}^{3}$.

\section{Classification of singular Cubic surfaces}

The classification of singular cubic surfaces in $\mathbf{P}^{3}$ given by J. W. Bruce and C. T. C. Wall [5] corresponds to another one in accordance with the incidence relations of the lines contained in a cubic surface, studied by several authors [6], 25]. A cubic surface is non-singular if and only if it contains 27 different lines. When it is singular, some of the lines collapse. The surface given by a general point of each stratum presents a certain type of coincidence between these lines.

We recall here the main points of Bruce's and Wall's work. If $P$ is a singular point of a cubic surface $C$ and $\left(x_{0}: x_{1}: x_{2}: x_{3}\right)$ are homogeneous coordinates in $\mathbf{P}^{3}$, one can assume, doing a change of coordinates if necessary, that $P$ is the point $(0: 0: 0: 1)$ and then, the equation of the surface takes the form

$$
x_{3} f_{2}\left(x_{0}, x_{1}, x_{2}\right)+f_{3}\left(x_{0}, x_{1}, x_{2}\right)=0
$$

where $f_{i}$ is homogeneous of degree $i$. The classification depends, first on the rank of the quadratic form $f_{2}$, and second, on the coincidences between the collection of 6 lines common to the two cones given by $f_{2}=0$ and $f_{3}=0$. These are lines through $P$, contained in $C$ and they are equivalent to the intersection points of the two plane curves, let's say $V_{2}$ and $V_{3}$, given by $f_{2}\left(x_{0}, x_{1}, x_{2}\right)=0$ and $f_{3}\left(x_{0}, x_{1}, x_{2}\right)=0$ respectively.

Let us suppose that the surface is irreducible and has only isolated singularities. According to the rank of $f_{2}$ there are 4 types of singularity at the fixed point $P$ : conic node, binode, uninode and triple point.

When the rank of $f_{2}$ is maximum, the singular point is a conic node. There are 11 strata in accordance with the partition of 6 given by the coincidences of the intersection points (lines) of $V_{2}$ and $V_{3}$, namely 


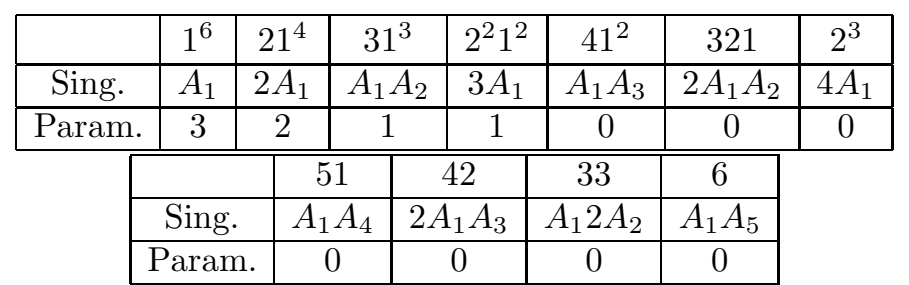

In this table, the partition called, for example, $2^{2} 1^{2}$ corresponds to a collection of 6 points with 2 of them being double points. At a singular point of type $A_{k}$, the normal form of the surface's equation is $x_{0}^{k+1}+\sum_{j=1}^{3} x_{j}^{2}=0$. The third row indicates the number of parameters on which each type of surface depends, up to projective transformations.

A binode corresponds to $\operatorname{rank}\left(f_{2}\right)=2$, in this case, $P$ is a singularity of type $A_{k}$ with $k \geq 2$. There are 13 possible configurations of common points to the cubic and the line pair and 6 of them correspond to new strata.

\begin{tabular}{|c|c|c|c|c|c|c|c|c|}
\hline & $1^{3} \cdot 1^{3}$ & $1^{3} .21$ & $1^{3} .3$ & 21.21 & & 1.3 & 3.3 & $1^{2} .1$ \\
\hline Sing. & $A_{2}$ & $\overline{A_{2} A_{1}}$ & $2 A_{2}$ & $\overrightarrow{A_{2} 2 A}$ & & ${ }_{2} A_{1}$ & $3 A_{2}$ & $A_{3}$ \\
\hline Param & 2 & 1 & 1 & 0 & & ) & 0 & 1 \\
\hline & & $1^{2} .2$ & 2.2 & $1^{2} .1$ & 2.1 & $21^{2} .0$ & 2 . & \\
\hline & & $A_{3} A_{1}$ & $A_{3} 2 A_{1}$ & $A_{4}$ & $A_{4} A_{1}$ & $A_{5}$ & $A_{5}$ & \\
\hline$\overline{\mathrm{Pa}}$ & $\mathrm{m}$. & $\overline{0}$ & $\overline{0}$ & $\overline{0}$ & $\overline{0}$ & $\overline{0}$ & 0 & \\
\hline
\end{tabular}

When $\operatorname{rank}\left(f_{2}\right)=1, P$ is a uninode and one gets three more strata called $D_{4}, D_{5}$ and $E_{6}$ corresponding to the cases in which the points in $V_{2} \cap V_{3}$ are three distinct points, two and one, respectively.

Finally, in the case of $f_{2}=0, P$ is a triple point. It must happen that $f_{3}=0$ defines a non-singular cubic in order to have an isolated singularity at $P$. The surface is then a cone over this plane cubic.

Until now the singular point $P$ is fixed on the cubic surface. When $P$ moves in $\mathbf{P}^{3}$, one gets strata in $\mathbf{P}^{19} \times \mathbf{P}^{3}$ of cubic surfaces with a distinguished singular point. Its projection onto the first factor gives the strata of singular cubic surfaces, living in $\mathbf{P}^{19}$, that we are interested in.

\section{PARAmeter SPACES}

We first construct a subscheme of $\mathbf{P}^{19} \times \mathbf{P}^{3}$, that we will call $S$, parametrizing cubic surfaces in $\mathbf{P}^{3}$ with a distinguished singular point.

In this way, strata of cubic surfaces with a singular point can be seen as subschemes of $S$ and the degree of corresponding strata of singular cubic surfaces in $\mathbf{P}^{19}$, obtained by projection onto the first factor, could be computed, using the projection formula (see 3.2.(c) of [9]), as a product in the Chow ring $A^{*}(S)$.

3.1. Cubics with a distinguished singular point. The construction of the parameter space $S$ for cubic surfaces with a distinguished singular point is analogous to others appearing in the literature for plane curves (see, for instance, [22] and [23]).

To fix notation, let $V$ be a vector space of $\operatorname{dim} 4$ over $\mathbf{C}$ and $\mathbf{P}^{3}=P(V)$ the projective space of lines of $V$. We set

$$
\left.0 \longrightarrow L \longrightarrow V\right|_{\mathbf{P}^{3}} \longrightarrow Q \longrightarrow 0
$$


for the tautological exact sequence with $\operatorname{rank}(L)=1$ and $\operatorname{rank}(Q)=3$ and we denote by $h=c_{1}\left(L^{*}\right)$ the first Chern class of $L^{*}=\mathcal{O}_{\mathbf{P}^{3}}(1)$ ( $h$ is the hyperplane class of $\mathbf{P}^{3}$ ) and by $S^{3} V^{*}$ the third symmetric power of $V^{*}$.

Definition 3.1. Let $F$ be the subbundle of $S^{3} V^{*} \mid \mathbf{P}^{3}$ whose fiber over a point of $\mathbf{P}^{3}$ is the linear subspace of $S^{3} V^{*}$ of cubic forms that have multiplicity at least 2 at such point. Denote by $S$ the projective bundle associated to $F$.

With this definition $S=P(F) \subset P\left(\left.S^{3} V^{*}\right|_{\mathbf{P}^{3}}\right) \cong \mathbf{P}^{19} \times \mathbf{P}^{3}$ is the incidence subvariety whose points consist in couples $(f, P)$, where $P$ is at least a double point for the cubic surface given by $f=0$. Since $\operatorname{rank}(F)=16$, then $\operatorname{dim}(S)=18$. In order to determine the intersection ring of $S=P(F)$, we start with a preliminary lemma.

Lemma 3.2. With the previous notation, the sequence of vector bundles over $\mathbf{P}^{3}$

$$
\left.0 \rightarrow S^{2} Q^{*} \otimes Q^{*} \stackrel{u}{\longrightarrow} S^{2} Q^{*} \otimes V^{*}\right|_{\mathbf{P}^{3}} \oplus S^{3} Q^{*} \stackrel{v}{\longrightarrow} F \rightarrow 0
$$

where $u(\alpha \otimes \beta)=(\alpha \otimes \beta,-\alpha \beta)$ and $v(\alpha \otimes \omega, \beta)=\alpha \omega+\beta$, is exact. Therefore $c(F)=1-8 h+40 h^{2}-160 h^{3}$.

Proof. The first part is similar to the analogous result for plane curves (see [22]). The second one is a straightforward computation applying Whitney's formula (see 3.2.(e) of [9]) to this resolution and to the tautological exact sequence over $\mathbf{P}^{3}$ above (3.1).

Proposition 3.3. Let $\pi$ be the projection from $S$ to $\mathbf{P}^{3}$. The intersection ring of $S=P(F)$ is

$$
A^{*}(P(F)) \cong \frac{\mathbf{Z}[b, \mu]}{\left(b^{4}, \mu^{16}-8 b \mu^{15}+40 b^{2} \mu^{14}-160 b^{3} \mu^{13}\right)}
$$

where $b=\pi^{*} h$ and $\mu=c_{1}\left(\mathcal{O}_{P(F)}(1)\right)$. Moreover, the following relations hold:

$$
b^{3} \mu^{15}=1, b^{2} \mu^{16}=8, b \mu^{17}=24 \text { and } \mu^{18}=32 .
$$

Proof. The description of $A^{*}(S)$ as a polynomial ring is a direct consequence of the result giving the Chow ring of a projective bundle $P(E)$ in terms of the total Chern class of $E$ and the intersection ring of the base space (see 3.3.(b) and Ex. 8.3.4. of 9]). In order to verify the relations in $A^{*}(S)$ we take down the products in this ring to $A^{*}\left(\mathbf{P}^{3}\right)$, using the projection formula and standard results about Chern classes (see Ex. 3.2.1. of [9]).

3.2. More parameter spaces. We build now a space, say $X$, that fixes a singular point $P$ for each cubic surface $C$, and parametrizes the lines contained in $C$ and passing through $P$. The coincidences between these lines determine the stratum where the surface lives.

To get $X$, we first construct another space $Z$ in $\mathbf{P}^{19} \times \mathbf{P}^{3} \times G r$. This $Z$ turns out to be a projective bundle over $S$ and we will define $X$ as a subscheme of $Z$. If we write $f$ for the restriction to $X$ of the projection from $Z$ to $S$, it is natural to consider the stationary multiple points of $f$ to solve our problem concerning the strata of singular cubic surfaces, because the condition defining the strata of cubics is the collapse produced between the points in the fiber of $X$ over $S$. 
Remark 3.4. One has to take care of three pairs of strata such that both strata on each pair have the same codimension in the $\mathbf{P}^{19}$ of cubic surfaces and they correspond to the same type of coincidence between points of $X$ over $S$. These pairs are $\left(4 A_{1}, D_{4}\right),\left(2 A_{1} A_{3}, D_{5}\right)$ and $\left(A_{1} A_{5}, E_{6}\right)$. In this paper, we don't compute the degree of the strata of surfaces having singularities of the types appearing on the two last pairs but, in order to compute the degree of surfaces with four conic nodes $\left(4 A_{1}\right)$ one must substract the contribution given by surfaces with a uninode $\left(D_{4}\right)$ (see Theorem 5.1).

3.2.1. Construction of $Z$. We consider the incidence of points and lines of $\mathbf{P}^{3}, I \subset$ $\mathbf{P}^{3} \times G r$, consisting of pairs $(P, l), P \in L$, where $l$ is the point of $G r$ corresponding to the line $L \subset \mathbf{P}^{3}$.

Definition 3.5. If $p_{1}$ (resp. $p_{2}$ ) is the projection from $I$ to $\mathbf{P}^{3}$ (resp. Gr), $Z$ is defined as the scheme $I \times \mathbf{P}^{3} S$ (see B.2.3. of [9]).

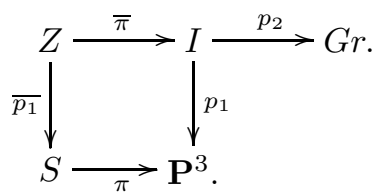

This $Z$ represents collections $\left(f_{C}, P, l\right)$ with $f_{C}=0$ the equation of the cubic surface $C, P$ a double point of $C$ and $L$ a line through $P$. By definition, $Z$ is a projective bundle over $S$ : the projection $p_{1}$ identifies the incidence $I$ with $P\left(T_{\mathbf{P}^{3}}\right)$, ( $T_{\mathbf{P}^{3}}$ is the tangent bundle of $\mathbf{P}^{3}$ ), so we have also (see B.5.5. of [9])

$$
I \cong P\left(T_{\mathbf{P}^{3}}(-2)\right) \cong P(Q(-1))
$$

and therefore $Z \cong P\left(\pi^{*} Q(-1)\right)$ and $\operatorname{dim}(Z)=20$.

It is also easy to see that $p_{2}^{*} \mathcal{O}_{\mathrm{Gr}}(1) \cong \mathcal{O}_{\mathrm{P}(\mathrm{Q}(-1))}(1), p_{1}^{*} \mathcal{O}_{\mathrm{P}^{3}}(1) \cong \mathcal{O}_{P(U)}(1)$ and $p_{2}: I \rightarrow G r$ can be identified with the projective bundle $P(U)$ over $G r$, being $U$ the tautological subbundle of rank 2 fitting in the tautological exact sequence over Gr:

$$
\left.0 \longrightarrow U \longrightarrow V\right|_{G r} \longrightarrow Q_{G r} \longrightarrow 0 \text {. }
$$

These facts will be useful on later computations (see 3.2.2).

3.2.2. Construction of $X$. If $C$ is a cubic surface in $\mathbf{P}^{3}$ given as the zeroes of a section $f_{C}$ of $\mathcal{O}_{\mathbf{P}^{3}}(3)$, the Fano scheme of lines contained in $C, F(C)$, can be seen as the scheme of zeroes of the section induced by $f_{C}$ in $p_{2 *}\left(p_{1}^{*} \mathcal{O}_{\mathbf{P}^{3}}(3)\right)=S^{3} U^{*}$. The line bundle $p_{1}^{*} \mathcal{O}_{\mathbf{P}^{3}}(3)$ over $I$ corresponds to the divisor $\left(p_{1}^{*} f_{C}\right)_{0}$ with fiber at a general $l \in G r$ the three intersection points of the cubic surface $C$ with the line $L$ of $\mathbf{P}^{3}$.

This is a particular case of the Fano scheme of pairs $(V, l)$ where $V$ is a hypersurface in $\mathbf{P}^{n}$ of given degree $d$ and $l$ parametrizes a line $L$ contained in $V$, defined in [1]. There it is proved that, if $H$ is the projective space of all hypersurfaces of degree $d, G$ is the grassmannian of lines of $\mathbf{P}^{n}$ and $U$ is the tautological subbundle over $G$, then the Fano scheme in $H \times G$ is given by the scheme of zeroes of the section of $S^{d} U^{*} \otimes \mathcal{O}_{H}(1)$, induced by the section of $\mathcal{O}_{\mathbf{P}^{n}}(d) \otimes \mathcal{O}_{H}(1)$ defining the universal divisor of $H \times \mathbf{P}^{n}$ (see Thm. 3.3. of [1]).

To obtain a space $X \subset Z$ consisting on the points $\left(f_{C}, P, l\right)$ of $Z$ such that $L \subset C$, we adapt this result to our case for which lines must be contained in the surface $C$ 
and must pass through the distinguished singular point $P \in C$ (see Proposition 3.9 below).

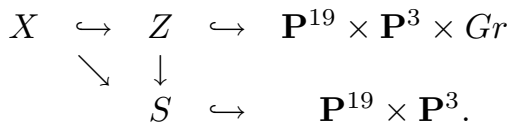

First, we define a space $T$ over $Z$ which plays the role of the incidence $I$ as projective bundle over $G r$ in the case of a concrete cubic surface: we want the fiber of $T$ over a point $\left(f_{C}, P, l\right)$ parametrized by $Z$ to represent the $\mathbf{P}^{1}$ of points of the line $L$. We then consider the diagram

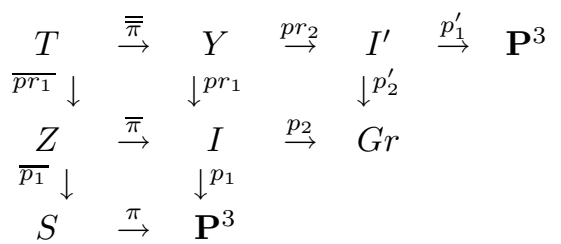

where $p_{2}^{\prime}$ is the projection of $P(U) \rightarrow G r, Y:=I \times_{G r} I^{\prime} \cong P\left(p_{2}^{*} U\right) \subset \mathbf{P}^{3} \times G r \times \mathbf{P}^{3}$ and $T:=Y \times{ }_{I} Z \cong P\left(\bar{\pi}^{*} p_{2}^{*} U\right) \subset \mathbf{P}^{19} \times \mathbf{P}^{3} \times G r \times \mathbf{P}^{3}$.

In this way, $Y$ parametrizes collections $\left(P, l, P^{\prime}\right)$ such that $P \in L$ and $P^{\prime} \in L$ and $T$ collections $\left(f_{C}, P, l, P^{\prime}\right)$ where $P$ is a double point of the surface $C$ and $P, P^{\prime} \in L$. Now, we build an invertible sheaf $N$ over $T$ which is the analogue of $p_{1}^{*} \mathcal{O}_{\mathbf{P}^{3}}(3)$ in the case of $F(C)$.

Lemma 3.6. Let $N$ be the line bundle $\overline{p r_{1}} * \bar{p}_{1} * \mathcal{O}_{S}(1) \otimes \mathcal{O}_{T}(3)$ over $T$. Then $N$ has an associated section $s_{N}$ such that $D:=\left(s_{N}\right)_{0}$ parametrizes collections $\left(f_{C}, P, l, P^{\prime}\right)$ with $P^{\prime} \in C$, besides $P$ being a double point of $C$ and $P, P^{\prime} \in L$.

Proof. Let $\pi_{1}$ and $\pi_{2}$ be the projections from $S \times \mathbf{P}^{3}$ to its factors. If $\phi$ is the morphism from $T$ to $S \times \mathbf{P}^{3}$ given by the diagram (3.4) then, by construction, $N$ is the pullback by $\phi$ of $\pi_{1}^{*} \mathcal{O}_{S}(1) \otimes \pi_{2}^{*} \mathcal{O}_{\mathbf{P}^{3}}(3)$. The section $s_{N}$ on the statement above is $\phi^{*}$ of the section of $\pi_{1}^{*} \mathcal{O}_{S}(1) \otimes \pi_{2}^{*} \mathcal{O}_{\mathbf{P}^{3}}(3)$ vanishing on the universal surface in $S \times \mathbf{P}^{3}$.

Remark 3.7. For each point $z \in Z$, the fiber of $\left(s_{N}\right)_{0} \subset T$ consists of two points: $P$ the double point of $C$ and, let's say $R$, the remaining point in the intersection of a general line $L$ through this $P$, and the surface $C$.

Let $D_{P}$ be the divisor of $T$ given by the point $P$ when $z$ moves in $Z$. Let $D_{R}$ be the residual divisor of $D_{P}$ on $D$ (see 9.2.1. of [9]), given by the point $R$ when $z$ moves in $Z$. We will get $X$ as the scheme of zeroes of a section of a rank 2-vector bundle over $Z$, defined as $\overline{p r}_{1}$ of the line bundle over $T$ corresponding to the divisor $D_{R}$.

Definition 3.8. Let $E$ be the sheaf $\overline{p r_{1}}\left(\mathcal{O}_{T}\left(D_{R}\right)\right)$, where $\overline{p r_{1}}$ is the projection from $T$ to $Z$ and $D_{R}$ is defined in the previous remark.

Proposition 3.9. With the previous notation:

1. The pairs consisting of a flat family of cubic surfaces of $\mathbf{P}^{3}$ with a distinguished singular point and a flat family of lines contained in the surface and passing through its singular point, both parametrized by the same scheme $W$ are the $W$-points of a closed subscheme $X$ of $Z$. 
2. The space $X$ is the scheme of zeroes of a regular section, say $s_{E}$, of the locally free sheaf $E$ of rank 2 over $Z$.

Proof. The proof is analogous to that of Thm. $3.3($ i), (iii) of [1] except for the following concerning 2 :

By definition, $D=2 D_{P}+D_{R}$, so $M:=\mathcal{O}_{T}\left(D_{R}\right)=N \otimes \mathcal{O}_{T}\left(-2 D_{P}\right)$ and the section $s_{N}$ of $N$ induces another one of $M$, let's say $s_{M}$, vanishing on $D_{R}$. The sought section is equal to $s_{E}=\overline{p r}_{1} s_{M}$.

$E$ is a rank 2-vector bundle because by Grauert's theorem (see III.12.9 of [12]), $E=R^{0} \overline{p r}_{1_{*}}\left(\mathcal{O}_{T}\left(D_{R}\right)\right)$ is locally free if $\operatorname{dim} H^{0}\left(T_{z},\left.\mathcal{O}_{T}\left(D_{R}\right)\right|_{T_{z}}\right)$ is constant $\forall z \in Z$ and in our case, $h^{0}\left(T_{z},\left.\mathcal{O}_{T}\left(D_{R}\right)\right|_{T_{z}}\right)=h^{0}\left(\mathbf{P}^{1}, \mathcal{O}_{\mathbf{P}^{1}}(1)\right)=2, \forall z \in Z$.

We write $j$ for the inclusion $X \subset Z, p$ for the projection from $Z$ to $S$ (called before $\overline{p_{1}}$ ) and $f$ for the composition $p \circ j$.

The situation we get is

$$
\begin{aligned}
& \begin{array}{c}
E \\
\downarrow \uparrow^{s_{E}}
\end{array} \\
& \begin{array}{rllll}
X=\left(s_{E}\right)_{0} & \stackrel{\searrow^{f}}{\hookrightarrow} & \begin{array}{l}
Z \\
\downarrow^{p}
\end{array} & \hookrightarrow & \mathbf{P}^{19} \times \mathbf{P}^{3} \times G r \\
& & \hookrightarrow & \mathbf{P}^{19} \times \mathbf{P}^{3}
\end{array}
\end{aligned}
$$

with the space $X$ parametrizing, for each cubic surface with a given double point, the lines passing through that point and contained in the surface. In this way, the strata of singular cubic surfaces with a conic node of given type (see \$2) depend on the coincidences between points on the fiber of $f: X \rightarrow S$, keeping in mind Remark 3.4. We will show in $\$ 5$ that their degree can be determined using a suitable cycle of stationary multiple points of $f$ and computing in the intersection ring of the space of 6-tuples $Z_{6}$ (see $\$ 4$ ).

In 95 we will need to know $c(E)$, the total Chern class of the vector bundle $E$ and also that $X$ is smooth.

Lemma 3.10. With the previous notation, if $\xi=c_{1}\left(\mathcal{O}_{Z}(1)\right)$ is the hyperplane class of $Z$ and we denote by $b, \mu$ the pullback by $p: Z \rightarrow S$ of the same classes in $A^{*}(S)$, then $E=\bar{\pi}^{*} p_{2}^{*} U^{*} \otimes \bar{p}_{1}^{*} \mathcal{O}_{S}(1) \otimes \bar{\pi}^{*} \mathcal{O}_{P(U)}(-2) \otimes \bar{\pi}^{*} \mathcal{O}_{P(Q(-1))}(2)$ and therefore $c(E)=1+(5 \xi-4 b+2 \mu)+\left(6 \xi^{2}+5 \mu \xi-9 b \xi+\mu^{2}-4 \mu b+3 b^{2}\right)$.

Proof. Since $E:=\overline{p r}_{1_{*}}\left(\mathcal{O}_{T}\left(D_{R}\right)\right)$ and $\mathcal{O}_{T}\left(D_{R}\right)=\mathcal{O}_{T}(D) \otimes \mathcal{O}_{T}\left(-2 D_{P}\right)$, we first determine $\left[D_{P}\right] \in A^{1}(T)$. If $\xi_{T}$ is the hyperplane class of $\left.T=P\left(\bar{\pi}^{*} p_{2}^{*} U\right)\right)$ over $Z$, then

$$
\left[D_{P}\right]=a \cdot \xi_{T}+\overline{p r}_{1}^{*}\left[D^{\prime}\right]
$$

with $D^{\prime} \in A^{1}(Z)$ (see Ex. 8.3.4. of $[9]$ ). Now, by construction, $\left[D_{P}\right] \cdot\left[T_{z}\right]=1$ and this implies $a=1$ (for $\xi_{T} \cdot\left[T_{z}\right]=1$ and the fibers of $T$ over $Z$ don't intersect each other).

To get $D^{\prime}$, we compute $\overline{p r}_{1_{*}}\left(\left[D_{P}\right] \cdot \xi_{T}\right)$ in two ways:

1. $\overline{p r_{1}}\left(\left[D_{P}\right] \cdot \xi_{T}\right)=\overline{p r}_{1 *}\left(\xi_{T}^{2}\right)+\left[D^{\prime}\right] \cdot \overline{p r}_{1 *}\left(\xi_{T}\right)=\bar{\pi}^{*} p_{2}^{*} c_{1}\left(Q_{G r}\right)+\left[D^{\prime}\right]$, where the first equality is the projection formula and the second is Ex. 3.2.1 of [9], being $Q_{G r}$ the tautological quotient of rank 2 over the grassmannian.

2. Let $s_{P}$ be the section of $T$ over $Z$ such that $\operatorname{Im}\left(s_{P}\right)=D_{P}$. Then

$$
\overline{p r}_{1_{*}}\left(\left[D_{P}\right] \cdot \xi_{T}\right)=c_{1}\left(s_{P}^{*} \mathcal{O}_{T}(1)\right) .
$$


From 1 and 2 above, $\left[D^{\prime}\right]=c_{1}\left(s_{P}^{*} \mathcal{O}_{T}(1)\right)-\bar{\pi}^{*} c_{1}\left(\mathcal{O}_{P(Q(-1))}(1)\right) \in A^{1}(T)$, recalling that $c_{1}\left(Q_{G r}\right)=c_{1}\left(\mathcal{O}_{G r}(1)\right)$ and $p_{2}^{*} \mathcal{O}_{G r}(1)=\mathcal{O}_{P(Q(-1))}(1)$.

To compute $c_{1}\left(s_{P}^{*} \mathcal{O}_{T}(1)\right)$, we use the upper part of diagram (3.4):

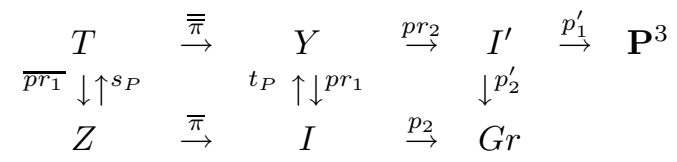

where we define $t_{P}$ as the section of $Y=I \times{ }_{G r} I^{\prime}$ whose image is the diagonal of $Y$. Looking at the diagram we see that $s_{P}^{*} \mathcal{O}_{T}(1)=\bar{\pi}^{*} t_{P}^{*} \mathcal{O}_{Y}(1)\left(\right.$ as $\left.T=P\left(\bar{\pi}^{*} p_{2}^{*} U\right)\right)$. Now, by construction, $t_{P}^{*} c_{1}\left(\mathcal{O}_{Y}(1)\right)=c_{1}\left(\mathcal{O}_{I}(1)\right)$ with $I=P(U)$ over $G r$.

Putting all these things together $\left[D^{\prime}\right]=\bar{\pi}^{*}\left(c_{1}\left(\mathcal{O}_{I}(1)\right)-c_{1}\left(\mathcal{O}_{P(Q(-1))}(1)\right)\right)$ and:

- $\mathcal{O}_{T}\left(D_{P}\right)=\mathcal{O}_{T}(1) \otimes \overline{p r}_{1}^{*} \bar{\pi}^{*} \mathcal{O}_{P(U)}(1) \otimes \overline{p r}_{1}^{*} \bar{\pi}^{*} \mathcal{O}_{P(Q(-1))}(-1)$,

- $\mathcal{O}_{T}\left(D_{R}\right)=\mathcal{O}_{T}(1) \otimes \overline{p r}_{1}^{*}\left(\bar{p}_{1}^{*} \mathcal{O}_{S}(1) \otimes \bar{\pi}^{*} \mathcal{O}_{P(U)}(-2) \otimes \bar{\pi}^{*} \mathcal{O}_{P(Q(-1))}(2)\right)$.

Finally, by the projection formula:

$$
E=\overline{p r}_{1_{*}}\left(\mathcal{O}_{T}\left(D_{R}\right)\right)=\bar{\pi}^{*} p_{2}^{*} U^{*} \otimes \bar{p}_{1}^{*} \mathcal{O}_{S}(1) \otimes \bar{\pi}^{*} \mathcal{O}_{P(U)}(-2) \otimes \bar{\pi}^{*} \mathcal{O}_{P(Q(-1))}(2) .
$$

From this expression, the computation of $c(E)$ is straightforward.

We see now that $X$ is smooth (even though $X$ is not smooth over $S$ ).

Proposition 3.11. With the previous notation, $X$ is smooth.

Proof. We are going to verify that, if $P \in \mathbf{P}^{3}$, then the fiber $X_{P}$ can be identified with the total space of a projective bundle over $\mathbf{P}^{2}$ and therefore $X$ is smooth.

Since $X=\left(s_{E}\right)_{0}$ with $s_{E}$ a section of the vector bundle $E$ over $Z$, then $X_{P} \subset Z_{P}$ can be identified with the scheme of zeroes of the section of $\left.E\right|_{Z_{P}}$ induced by $s_{E}$. Now $Z_{P} \cong \mathbf{P}^{15} \times \mathbf{P}^{2}$ and, if $\tau_{1}$ y $\tau_{2}$ are the projections from $\mathbf{P}^{15} \times \mathbf{P}^{2}$ to its factors, then

$$
\left.E\right|_{Z_{P}}=\tau_{1}^{*} \mathcal{O}_{\mathbf{P}^{15}}(1) \otimes \tau_{2}^{*}\left(\mathcal{O}_{\mathbf{P}^{2}}(2) \oplus \mathcal{O}_{\mathbf{P}^{2}}(3)\right) .
$$

To see this we use the explicit description of $E$ given in the previous lemma, noting that:

- The fiber $I_{P}$ consists on the plane in $G r$ of lines through the point $P$.

- $\left.p_{2}^{*} U^{*}\right|_{I_{P}} \cong \mathcal{O}_{\mathbf{P}^{2}} \oplus \mathcal{O}_{\mathbf{P}^{2}}(1)$. This can be checked recalling that

$$
U^{*} \cong p_{2 *}\left(p_{1}^{*} \mathcal{O}_{\mathbf{P}^{3}}(1)\right),
$$

and so, every section $\rho$ of $U^{*}$ is induced by a section $\eta$ of $\mathcal{O}_{\mathbf{P}^{3}}(1)$ in such a way that $\rho$ vanishes on the plane $\Pi_{\Lambda}$ of $G r$ parametrizing the lines contained in the plane $\Lambda$ which is the zero locus of $\eta$. In this way, the restriction of the Koszul exact sequence (see B.3.4. of [9]):

$$
0 \rightarrow \mathcal{O}_{G r} \rightarrow U^{*} \rightarrow \mathcal{I}_{\Pi_{\Lambda}}(1) \rightarrow 0
$$

to $I_{P}$ is

$$
\left.0 \rightarrow \mathcal{O}_{\mathbf{P}^{2}} \rightarrow U^{*}\right|_{\mathbf{P}^{2}} \rightarrow \mathcal{O}_{\mathbf{P}^{2}}(1) \rightarrow 0,
$$

for the intersection in $G r$ of a plane of lines through a general point of $\mathbf{P}^{3}$ and a plane of lines contained in a general plane of $\mathbf{P}^{3}$ is empty (see Ex. 14.7.2. of [9]).

- $\left.\bar{p}_{1}^{*} \mathcal{O}_{S}(1)\right|_{Z_{P}} \cong \mathcal{O}_{\mathbf{P}^{15}}(1)$ (recalling that $\left.S_{P} \cong \mathbf{P}^{15}\right)$. 
- The restriction of $\mathcal{O}_{P(U)}(-1)$ to $I_{P}$ is trivial since $I \cong P(U) \stackrel{p_{2}}{\longrightarrow} G r$ and

$$
\mathcal{O}_{P(U)}(1)=p_{1}^{*} \mathcal{O}_{\mathbf{P}^{3}}(1) \text {. }
$$

- $\left.\mathcal{O}_{P(Q(-1))}(2)\right|_{I_{P}}=\mathcal{O}_{\mathbf{P}^{2}}(2)$, for $I \cong P(Q(-1)) \stackrel{p_{1}}{\rightarrow} \mathbf{P}^{3}$.

Now, if we call $R:=\mathcal{O}_{\mathbf{P}^{2}}(2) \oplus \mathcal{O}_{\mathbf{P}^{2}}(3)$, then $P\left(H^{0}(R)\right) \cong \mathbf{P}^{15}$ and the sequence of vector bundles over $\mathbf{P}^{2}$ :

$$
0 \rightarrow K \rightarrow H^{0}(R) \otimes \mathcal{O}_{\mathbf{P}^{2}} \stackrel{e v}{\rightarrow} R \rightarrow 0,
$$

given by the evaluation of sections of $R$, is exact. The key observation is that the tautological section $\Gamma$ of $\tau_{1}^{*} \mathcal{O}_{\mathbf{P}^{15}}(1) \otimes \tau_{2}^{*} R=\left.E\right|_{Z_{P}}$ is precisely the restriction to the fiber $Z_{P}$ of $s_{E}$.

The situation is

$$
\begin{aligned}
& \begin{array}{l}
\tau_{1}^{*} \mathcal{O}_{\mathbf{P}^{15}}(1) \otimes \tau_{2}^{*} R \\
\downarrow \uparrow \Gamma
\end{array} \\
& (\Gamma)_{0} \\
& \begin{aligned}
\searrow & P\left(H^{0}(R)\right) \times \mathbf{P}^{2} \\
& \downarrow \\
& P\left(H^{0}(R)\right)
\end{aligned}
\end{aligned}
$$

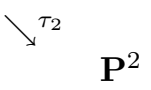

and so $X_{P} \cong(\Gamma)_{0}$ which is isomorphic to the projective bundle $P(K)$ over $\mathbf{P}^{2}$. Therefore $X$ is smooth.

\section{SPACES FOR $k$-TUPLES AND SECANT BUNDLES}

In $\S 5$ we shall use the spaces for $k$-tuples $Z_{k}$ and the secant bundles $E_{k}$, for $Z$ and $E$ defined in 93 , to compute the degree of some strata of cubic surfaces with a conic node. We describe them here taking as reference the work by Z. Ran 24].

Spaces for $k$-tuples. The space $Z_{k}$ is built inductively as a suitable blow-up of an ordered $k$-product of $Z$.

- Steps 0,1 . One denotes $Z_{0}:=S, Z_{1}:=Z, p_{0}^{1}: Z_{1} \rightarrow Z_{0}$ is the morphism $p$ and one defines $\iota_{j}: Z_{j} \rightarrow Z_{j}$ as the identity $i$ for $j=0,1$.

- Step 2. Considering the fiber product defined by the cartesian diagram (see B.2.3. of $[9])$ :

$$
\begin{array}{ccc}
Z_{1} \times Z_{0} Z_{1} & \stackrel{p r_{2}}{\rightarrow} & Z_{1} \\
\downarrow^{p_{1}} & & \downarrow^{p} \\
Z_{1} & \stackrel{p}{\rightarrow} & Z_{0},
\end{array}
$$

one defines a diagonal $\Delta_{1}=\left(i \times \iota_{1}\right)\left(Z_{1}\right) \hookrightarrow Z_{1} \times Z_{0} Z_{1} ; b_{2}$, the blow-up of $Z_{1} \times Z_{0} Z_{1}$ along $\Delta_{1}$ with exceptional divisor $D_{1,2}^{2}$,

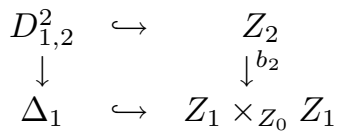

and new projections from $Z_{2}$ to $Z_{1} ; p_{1,1}^{2}:=p r_{1} \circ b_{2}$ y $p_{2,2}^{2}:=p r_{2} \circ b_{2}$.

Since $p$ is smooth, $\Delta_{1} \hookrightarrow Z_{1} \times_{Z_{0}} Z_{1}$ is a regular inclusion (see B.7.3. of [9]) and the new projections are also smooth.

Out of the exceptional divisor, $Z_{2}$ parametrizes couples $\left(z_{1}, z_{2}\right)$ of different points of $Z$ having the same image in $S$, i.e., strict double points of $f$. In $D_{1,2}^{2}$ live the couples of points of $Z$ having the same image by $p$ that are infinitely near $\left(b_{2}\left(D_{1,2}^{2}\right)\right.$ consists on pairs $(z, z)$ where $z$ is a ramification point of $\left.p\right)$. 
One notes that $\left(\iota_{1} \circ p r_{2, \iota_{1}} \circ p r_{1}\right)$ is an involution of $Z_{1} \times Z_{0} Z_{1}$ reversing the order in $\left(z_{1}, z_{2}\right)$ and fixing the diagonal $\Delta_{1}$, so it lifts to an involution of $Z_{2}$ that will be denoted by $\iota_{2}$.

- Induction hypothesis. One supposes that a space $Z_{k}$ of $k$-tuples and associated objects $\left(D_{1, k}^{k}, p_{1, k-1}^{k}, p_{2, k}^{k}, \iota_{k}\right)$ are built as before.

- Step $k+1$. One considers the fiber product defined by

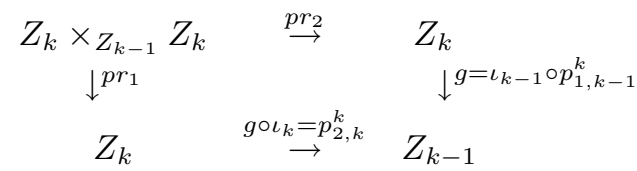

where $p_{1, k-1}^{k}:=p r_{1} \circ b_{k}, p_{2, k}^{k}:=p r_{2} \circ b_{k}$ and $b_{k}: Z_{k} \rightarrow Z_{k-1} \times_{Z_{k-2}} Z_{k-1}$ is the previous blow-up.

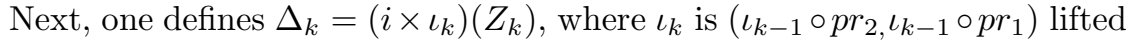
to $Z_{k}$ ( $\iota_{k}$ is a regular inclusion by B.7.3. of [9]) and the blow-up along $\Delta_{k}$ with exceptional divisor $D_{1, k+1}^{k+1}, b_{k+1}: Z_{k+1} \rightarrow Z_{k} \times Z_{k-1} Z_{k}$.

In this way, the points of $Z_{k}$ correspond to ordered $k$-tuples of points of $Z$ having the same image by $p$ in $S$. By B.7.3. of [9, it holds

$$
N_{\Delta_{k}} Z_{k} \times Z_{k-1} Z_{k} \cong \iota_{k}^{*} T_{\iota_{k-1} \circ p_{1, k-1}^{k}} \cong T_{p_{2, k}^{k}} .
$$

This will be useful in $\sqrt{6}$, in order to make computations in the intersection ring $A^{*}\left(Z_{k}\right)$.

Remark 4.1. Note that in [24] $g$ is taken as $p_{1, k-1}^{k}$ and so $\Delta_{k}=\left(i \times \iota_{k}\right)\left(Z_{k}\right)$ is not contained in $Z_{k} \times Z_{k-1} Z_{k}$.

Regarding the enumeration of stationary multiple points of the morphism $p$, given a partition $\sigma=\left(k_{1}, k_{2}, \ldots, k_{r}\right)$ of $k$ with $\sum_{i=1}^{r} k_{i}=k$, Ran defines a subscheme of $Z_{k}$ called $V_{\sigma}$, parametrizing $k$-tuples of $Z / S$ with the first $k_{1}$ points being infinitely near to each other (i.n.), the next $k_{2}$ points being also i.n., etc. For example, the subscheme of $Z_{4}$ parametrizing pairs of points of type $(2,2)$ is $V_{(2,2)}=\left(p_{1,2}^{3} \circ p_{1,3}^{4}\right)^{*} D_{1,2}^{2} \cap\left(p_{2,4}^{4}\right)^{*} D_{1,3}^{3}$ for points in $Z_{4}$ correspond to collections $\left(z_{1}, z_{2}, z_{2}, z_{3}, z_{3}, z_{2}, z_{2}, z_{4}\right)$ of $Z / S$.

Anyway, for each partition $\sigma$ of $k$, one can define a subscheme of $Z_{k}$ parametrizing $k$-tuples of type $\sigma$ as a suitable intersection of (pullbacks by projections of) exceptional divisors reflecting the coincidences given by $\sigma$.

Remark 4.2. The space $Z_{k}$ had already been defined by S. L. Kleiman (see p. 390 of [16] and [17]). For the construction of $Z_{k+1}$, Kleiman considers the fiber product $Z_{k} \times Z_{k-1} Z_{k}$ containing the usual diagonal $(i \times i)\left(Z_{k}\right)$.

For the justification of $Z_{k}$ as parameter spaces, see [18 and 24] where a relation between $Z_{k}$ and the relative Hilbert scheme $\operatorname{Hilb}^{k}(Z / S)$ is established, showing that $Z_{k}$ is a parameter space for ordered and curvilinear $k$-tuples of points of $Z$ with the same image in $S$ (a collection of points is curvilinear if the points live in a smooth curve). Consequently, the (stationary) multiple points formulas given by Colley, Kleiman and Ran cannot enumerate the non-curvilinear points of a morphism.

Secant bundles. The notion of secant bundle, introduced by R. L. E. Schwarzenberger [27] in 1964, generalizes the concept of tangent bundle of a projective variety $Y$ in the following sense: the secant bundle of $Y$ is defined over a product scheme of $Y$ so that its restriction to the diagonal in this product is the tangent bundle of 
$Y$. The Chern classes of secant bundles are related with multiple-point cycles (see [21], [19] and [29]). By definition, a secant bundle is always associated to a line bundle over a scheme.

In [24], given a vector bundle $E$ of arbitrary rank over a smooth scheme $Z$, a secant bundle $E_{k}$ over $Z_{k}$ is defined, allowing us to compute the class $\left[X_{k}\right]$ in $A^{*}\left(Z_{k}\right)$, when $X$ is the zero subscheme of a section of $E$. The $E_{k}$ are defined inductively and they fit into an exact sequence of vector bundles over $Z_{k}$ (analogous to the one given by Schwarzenberger for its secant bundles) from which one can compute its Chern classes.

Regarding the definition of $E_{k}$ : for $k=1, E_{1}:=E$; if $k=2, E_{2}$ is defined by the exact sequence over $Z_{2}$ :

$$
\left.0 \rightarrow E_{2} \stackrel{\left(\lambda_{2}^{1}, \lambda_{2}^{1}\right)}{\longrightarrow}\left(p_{1,1}^{2}\right)^{*} E \oplus\left(p_{2,2}^{2}\right)^{*} E \quad \stackrel{\rho_{2}}{\rightarrow}\left(p_{1,1}^{2}\right)^{*} E\right|_{D_{1,2}^{2}} \rightarrow 0,
$$

with $\rho_{2}(a, b)=\operatorname{res}(a)-r e s(b)$, and res the restriction to the exceptional divisor $D_{1,2}^{2}$. $E_{2}$ is a vector bundle over $Z_{2}$ by Nakayama's lemma and from its definition it is easy to see that

$$
0 \rightarrow\left(p_{2,2}^{2}\right)^{*} E \otimes L_{-D_{1,2}^{2}} \rightarrow E_{2} \rightarrow\left(p_{1,1}^{2}\right)^{*} E \quad \rightarrow \quad 0
$$

is exact, where in general $L_{D}$ is the line bundle associated to the divisor $D$.

When $k \geq 3$, by induction, one supposes that, $\forall j \leq k$, vector bundles $E_{j}$ and applications

$$
\left\{\begin{array}{c}
\lambda_{j}^{1}: E_{j} \rightarrow\left(\iota_{j-1} \circ p_{1, j-1}^{j}\right)^{*} E_{j-1} \\
\lambda_{j}^{2}: E_{j} \rightarrow\left(p_{2, j}^{j}\right)^{*} E_{j-1}
\end{array}\right\},
$$

are defined. Then

Definition 4.3. Let $J_{k+1}:=\left(\iota_{k} \circ p_{1, k}^{k+1}\right)^{*} E_{k} \oplus\left(p_{2, k+1}^{k+1}\right)^{*} E_{k}$ and let $K_{k+1}$ be the vector bundle given by the exact sequence over $Z_{k+1}$ :

$$
\left.0 \rightarrow K_{k+1} \stackrel{\left(\kappa^{1}, \kappa^{2}\right)}{\longrightarrow} J_{k+1} \stackrel{\rho_{k+1}}{\rightarrow}\left(p_{2, k+1}^{k+1}\right)^{*} E_{k}\right|_{D_{1, k+1}^{k+1}} \rightarrow 0,
$$

with $\rho_{k+1}(a, b):=\operatorname{res}(a)-\operatorname{res}(b)$. Let $E_{k+1}$ be defined by

$$
\left.0 \rightarrow E_{k+1} \stackrel{\left(\lambda_{k+1}^{1}, \lambda_{k+1}^{2}\right.}{\longrightarrow}\right) K_{k+1} \stackrel{\nu_{k+1}}{\longrightarrow}\left(p_{2, k}^{k+1}\right)^{*} E_{k-1} \otimes L_{-D_{1, k+1}^{k+1}} \rightarrow 0,
$$

with

$$
\begin{gathered}
\nu_{k+1}=\left(\left(\left(p_{1, k}^{k+1}\right)^{*}\left(\lambda_{k}^{2}\right)-\left(p_{2, k+1}^{k+1}\right)^{*}\left(\lambda_{k}^{1}\right)\right),\right. \\
p_{2, k}^{k+1}:=\iota_{k-1} \circ p_{1, k-1}^{k} \circ p_{2, k+1}^{k+1}: Z_{k+1} \rightarrow Z_{k-1} .
\end{gathered}
$$

A standard computation using commutative diagrams shows that with this definition the sequence of vector bundles over $Z_{k+1}$ :

$$
0 \rightarrow\left(p_{k+1, k+1}^{k+1}\right)^{*} E \otimes L_{-D^{k+1, k+1}} \quad \rightarrow E_{k+1} \rightarrow\left(p_{1, k}^{k+1}\right)^{*} E_{k} \quad \rightarrow 0
$$

where $p_{k+1, k+1}^{k+1}:=p_{2,2}^{2} \circ \cdots \circ p_{2, k}^{k} \circ p_{2, k+1}^{k+1}$ and

$$
D^{k+1, k+1}:=D_{1, k+1}^{k+1}+\left(p_{2, k+1}^{k+1}\right)^{*} D_{1, k}^{k}+\ldots+\left(p_{2,3}^{3} \circ p_{2,4}^{4} \circ p_{2,5}^{5} \circ \cdots \circ p_{2, k+1}^{k+1}\right)^{*} D_{1,2}^{2}
$$

is exact (for details see [30]). 
Remark 4.4. Note that our definition of $E_{k}$ for $k \geq 3$ is different from the one given in [24] for which, an easy computation, using Whitney's formula (3.2.(e) of 9]), shows that the total Chern class of $E_{k+1}$ is not the one given by the exact sequence (4.2) with which, in some examples, one recovers known results (see [30]).

\section{Degree of Some strata of CUbiC SURFACES With A CONIC NODE}

Let $\sigma=\left(k_{1}, k_{2}, \ldots, k_{r}\right)$ be a partition of 6 with $k_{j} \geq k_{l}$, if $j \leq l, C_{\sigma}$ the stratum in $\mathbf{P}^{19}$ of cubic surfaces with a conic node of type $\sigma, C_{U}$ the one of surfaces with a uninode and $\alpha_{\sigma}:=\operatorname{dim}\left(C_{\sigma}\right)$.

In principle, we would get $\operatorname{deg}\left(C_{\sigma}\right)$ as $\#\left(C_{\sigma} \cap \Lambda\right)$ being $\Lambda \subset \mathbf{P}^{19}$ a general linear space of codimension $\alpha_{\sigma}$.

The stratum $C_{\sigma}$ depends on coincidences between points on the fibers of the map $f: X \rightarrow S$, unless in the case of a coincidence of type $\sigma=(2,2,2)$ that corresponds to the union of $C_{(2,2,2)}$ and $C_{U}$ (see Remark 3.4). Since $X \subset Z$ is not smooth over $S$, we consider the space $Z_{6}$ of 6-tuples of $Z / S$ treated in $\$ 4$ Even though $f$ is not smooth, the inclusion $j: X \hookrightarrow Z$ induces, by construction, inclusions $j_{k}: X_{k} \hookrightarrow Z_{k}$.

Let $\phi_{1}$ denote the projection from $Z_{6}$ to $Z$ assigning to each 6-tuple of $Z_{6}$ its first element and $\tau$ the projection from $S$ to $\mathbf{P}^{19}$. Let $\phi$ be the composite projection $\tau \circ p \circ \phi_{1}: Z_{6} \rightarrow \mathbf{P}^{19}$ and $V_{\sigma}$ the subscheme of $Z_{6}$ corresponding to 6-tuples with a coincidence of type $\sigma$ (see $₫ 4$ ). A diagram of the situation is:

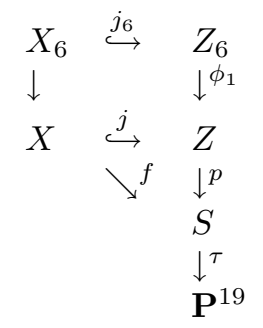

The result giving the degree of some strata of cubic surfaces with a conic node is the following

Theorem 5.1. Let $\sigma=\left(k_{1}, \ldots, k_{r}\right)$ be a partition of 6 with $r \geq 3$ and let $\Lambda$ be a linear space of $\mathbf{P}^{19}$ of dimension $7-r$. If $\Pi=\phi^{-1}(\Lambda)$, then the degree of the stratum $C_{\sigma}$ of cubic surfaces with a conic node of type $\sigma$ is

$$
\operatorname{deg}\left(C_{\sigma}\right)=\frac{1}{n(\sigma)} \int_{Z_{6}}\left[X_{6}\right] \cdot\left[V_{\sigma}\right] \cdot[\Pi],
$$

except for the case $\sigma=(2,2,2)$ where

$$
\operatorname{deg}\left(C_{\sigma}\right)=\frac{1}{n(\sigma)} \int_{Z_{6}}\left[X_{6}\right] \cdot\left[V_{\sigma}\right] \cdot[\Pi]-\frac{1}{4} \operatorname{deg}\left(C_{U}\right) .
$$

In both formulas, $n(\sigma)$ is an integer determined by the partition $\sigma$.

Proof. We divide the proof into 4 steps.

1. We first translate the computation of $\operatorname{deg}\left(C_{\sigma}\right)$ to $A^{*}(S)$ showing that, if $S_{\sigma} \subset$ $S$ is the closure of the locus of cubic surfaces with a distinguished singular point such that the associated collection of lines through this point have 
collapsed in accordance with the partition $\sigma$ (i.e. $\tau\left(S_{\sigma}\right)=C_{\sigma}$ ), then if $\sigma \neq$ $(2,2,2)$,

$$
m \cdot \operatorname{deg}\left(C_{\sigma}\right)=\int_{S}\left[S_{\sigma} \cap \Lambda_{S}\right]
$$

where $m$ is the number of conic nodes of a cubic surface in the stratum $C_{\sigma}$ and $\Lambda_{S}=\tau^{-1}(\Lambda)$ (see Lemma 5.2$)$.

When $\sigma=(2,2,2), \tau\left(S_{\sigma}\right)=C_{\sigma} \cup C_{U}$ and therefore

$$
4 \cdot \operatorname{deg}\left(C_{\sigma}\right)+\operatorname{deg}\left(C_{U}\right)=\int_{S}\left[S_{\sigma} \cap \Lambda_{S}\right] .
$$

2. We define a subscheme $W \subset Z_{6}$ by $W=X_{6} \cap V_{\sigma} \cap \Pi$ (in this way $\left(p \circ \phi_{1}\right)(W)=$ $\left.S_{\sigma} \cap \Lambda_{S}\right)$ and we prove that $[W] \in A^{*}\left(Z_{6}\right)$ enumerates the ordered 6-tuples of $\phi_{1}(W)$ over $S$ if and only if $\operatorname{dim}\left(\Lambda_{S}\right) \leq 3$, which is equivalent to $r \geq 3$ above (see Lemma 5.3). For the rest of the proof we then suppose that $\operatorname{dim}\left(\Lambda_{S}\right) \leq 3$.

3. We show in Lemma 5.4 that the cycle $[W] \in A^{*}\left(Z_{6}\right)$ pushes forward to $\widetilde{n}(\sigma)$. $\left[S_{\sigma} \cap \Lambda_{S}\right]$ in $A^{*}(S)$, and we compute $\widetilde{n}(\sigma)$ according to the partition $\sigma$.

4. We verify in Lemma 5.5 that $[W]=\left[X_{6} \cap V_{\sigma} \cap \Pi\right]=\left[X_{6}\right] \cdot\left[V_{\sigma}\right] \cdot[\Pi]$. In this way, the theorem is proved with $n(\sigma)=m \cdot \widetilde{n}(\sigma)$ depending only on the partition $\sigma$.

Lemma 5.2. With the previous notation, let $m$ be the number of conic nodes presented by a surface belonging to the stratum $C_{\sigma}$, then if $\sigma \neq(2,2,2)$,

$$
m \cdot \operatorname{deg}\left(C_{\sigma}\right)=\int_{S}\left[S_{\sigma} \cap \Lambda_{S}\right]
$$

and if $\sigma=(2,2,2)$,

$$
m \cdot \operatorname{deg}\left(C_{\sigma}\right)+\operatorname{deg}\left(C_{U}\right)=\int_{S}\left[S_{\sigma} \cap \Lambda_{S}\right]
$$

Proof. Suppose that $\sigma \neq(2,2,2)$ : if the type of singularities presented by a cubic surface corresponding to a point of $S_{\sigma}$ is $m A_{1} A_{q}$ (see $\left\{2\right.$ ) then $\operatorname{deg}\left(\left.\tau\right|_{S_{\sigma}}\right)=m$ (for the projection $\tau$ forgets the double point) and consequently $\tau_{*}\left(\left[S_{\sigma}\right]\right)=m \cdot\left[C_{\sigma}\right]$.

By construction, $\mu:=c_{1}\left(\mathcal{O}_{S}(1)\right)=\tau^{*} H$, where $H$ is the hyperplane class of $\mathbf{P}^{19}$. Applying the projection formula, we then have

$$
m \cdot \operatorname{deg}\left(C_{\sigma}\right)=m \int_{\mathbf{P}^{19}}\left[C_{\sigma}\right] \cdot H^{\alpha_{\sigma}}=\int_{S}\left[S_{\sigma}\right] \cdot \mu^{\alpha_{\sigma}}=\int_{S}\left[S_{\sigma} \cap \Lambda_{S}\right] .
$$

The last equality is $\left[S_{\sigma} \cap \Lambda_{S}\right]=\left[S_{\sigma}\right] \cdot\left[\Lambda_{S}\right]$. This is true because the intersection $S_{\sigma} \cap \Lambda_{S}$ consists of points and it is transversal at each one of them. (If this last claim were false, it would be also false for $\tau\left(S_{\sigma} \cap \Lambda_{S}\right)=C_{\sigma} \cap \Lambda$ and we know that a general linear space in a projective space has a non-empty intersection with any subvariety of complementary dimension and one can choose it so the intersection is transversal by Bertini's theorem (see II.8.18 of [12])).

If $\sigma=(2,2,2)$, a surface corresponding to a point of $S_{\sigma}$ can have 4 conic nodes or else a uninode, so this time $\tau_{*}\left(\left[S_{\sigma}\right]\right)=4 \cdot\left[C_{\sigma}\right]+\left[C_{U}\right]$ and the rest is as before.

With the notation given in 94 , if $p: Z \rightarrow S$, a cycle $[A] \in Z_{k}$ enumerates all the $k$-tuples of $\phi_{1}(A) \subset Z$ with the same image in $S$ if and only if $\left.p\right|_{\phi_{1}(A)}$ is curvilinear. We recall that a map $p: Z \rightarrow S$ is called curvilinear when $\operatorname{dim}\left(\Omega_{p}^{1}(z)\right) \leq 1, \forall z \in Z$ 
(with $\Omega_{p}^{1}$ the relative differentials (see B.2.7 of $[9]$ )) and that, if $\operatorname{dim}(Z)=n$, $\operatorname{dim}(S)=m$ and

$$
\overline{S_{i}}(p):=\{z \in Z: \operatorname{rango}(d p) \leq j\}=\left\{z \in Z: \operatorname{dim}\left(\Omega_{p}^{1}(z)\right) \geq n-j\right\},
$$

where $j=\min (n, m)-i$, is the locus of Thom-Boardman singularities of $p$ (see [16]), then if $n \leq m, p$ curvilinear $\Leftrightarrow \overline{S_{2}}(p)=\emptyset$, and if $n \geq m, p$ curvilinear implies that $\overline{S_{2}}(p)=\emptyset$.

Lemma 5.3. The cycle $[W]=\left[X_{6} \cap V_{\sigma} \cap \Pi\right]$ enumerates all the (ordered) 6-tuples of $\phi_{1}(W)$ with the same image in $S$ if and only if $\operatorname{dim}\left(\Lambda_{S}\right) \leq 3$.

Proof. Taking suitable scheme-theoretic inverse images of $X$ through the inductive construction of $X_{6}$ and then going down to $X$ by appropriated projections, one can define a subscheme $X_{\sigma} \subset X$ parametrizing the points $x \in X$ such that $\exists x_{1}, x_{2}, \ldots, x_{5} \in X$ with $f(x)=f\left(x_{1}\right)=\cdots=f\left(x_{5}\right)$, and the first $k_{1}$ points (included $x$ ) are i.n., the next $k_{2}$ points are i.n., and the last $k_{r}$ points are i.n., where i.n. means infinitely near points (see 2.3. of [7]).

Recalling the previous definitions, we have

$$
\phi_{1}(W)=j\left(X_{\sigma} \cap f^{-1} \Lambda_{S}\right) .
$$

Since $j$ is a regular inclusion and $f=p \circ j$, then $\left.p\right|_{\phi_{1}(W)}$ will be curvilinear when $\left.f\right|_{X_{\sigma} \cap f^{-1} \Lambda_{S}}$ is curvilinear. Now, $f$ curvilinear $\Leftrightarrow \overline{S_{2}}(f)=\emptyset$, for $\operatorname{dim}(X)=\operatorname{dim}(S)$. We then study the Thom-Boardman singularities' locus of $f$,

$$
\overline{S_{2}}(f)=\left\{x \in X: \operatorname{dim}\left(\Omega_{f}^{1}(x)\right) \geq 2\right\}=\left\{x \in X: r k\left(d f_{x}\right) \leq \operatorname{dim}(X)-2\right\},
$$

with $d f_{x}: T_{x} X \rightarrow T_{f(x)} S$, the map induced by $f$ on the tangent spaces.

By definition of $\overline{S_{2}}(f)$, we have $\operatorname{codim}\left(\overline{S_{2}}(f), X\right) \leq 2 \cdot 2=4$ (see (III, 5) of [16]). Checking that, in fact, for our $f$ this codimension is 4 , the proof is finished because in that case, when $\operatorname{dim}\left(\Lambda_{S}\right) \leq 3, f^{-1}\left(\Lambda_{S}\right)$ can be chosen so that $f^{-1}\left(\Lambda_{S}\right) \cap \overline{S_{2}}(f)=\emptyset$ and it is easy to see that $\operatorname{dim}\left(\Lambda_{S}\right) \leq 3 \Leftrightarrow r \geq 3$.

Let us verify that $\operatorname{codim}\left(\overline{S_{2}}(f), X\right)=4$. We recall from (2.1) that, fixed $P \in \mathbf{P}^{3}$, a cubic surface of $\mathbf{P}^{3}$ with $P$ singular, has equation $x_{3} f_{2}+f_{3}=0$, up to change of coordinates. The plane where the conic and cubic curves associated to $f_{2}$ and $f_{3}$, respectively, live is fixed and all possible surfaces correspond to all possible pairs of conic and cubic.

If $R=\mathcal{O}_{\mathbf{P}^{2}}(2) \oplus \mathcal{O}_{\mathbf{P}^{2}}(3)$ (see Proposition 3.11), then one gets $X_{P} \hookrightarrow P\left(H^{0}(R)\right) \times$ $\mathbf{P}^{2} \cong \mathbf{P}^{15} \times \mathbf{P}^{2}$ intersecting every pair of conic and cubic curves and, consequently, the part of $T_{x} X$ living in $T_{w} \mathbf{P}^{2}$, which eventually will contribute to the locus $\overline{S_{2}}(f)$ is the intersection of the Zariski tangent spaces of the two curves at the point $w$. In that way, $x \in \overline{S_{2}}(f)$ when this intersection has dimension 2 and so $\operatorname{codim}\left(\overline{S_{2}}(f), X\right)$ is the number of conditions for a pair of plane curves of degree 2 and 3 having an intersection of their tangent spaces at a common point of dimension 2. It is easy to see that this number is 4 (because that happens if and only if the two curves are singular at the common point).

Lemma 5.4. With the previous notation:

$$
\int_{Z_{6}}[W]=\int_{S} \widetilde{n}(\sigma) \cdot\left[S_{\sigma} \cap \Lambda_{S}\right]
$$


where $\widetilde{n}(\sigma)$ is an integer determined by the partition $\sigma$ according to the following table:

\begin{tabular}{|c|c|}
\hline$\sigma$ & $\widetilde{n}(\sigma)$ \\
\hline$(1,1,1,1,1,1)$ & $6 !=5 ! \cdot 6$ \\
\hline$(2,1,1,1,1)$ & $24=4 ! \cdot 1$ \\
\hline$(3,1,1,1)$ & $6=3 ! \cdot 1$ \\
\hline$(2,2,1,1)$ & $4=2 \cdot 2$ \\
\hline$(4,1,1)$ & $2=2 \cdot 1$ \\
\hline$(3,2,1)$ & 1 \\
\hline$(2,2,2)$ & $6=2 \cdot 3$ \\
\hline
\end{tabular}

Proof. By construction, $\left.p\right|_{\phi_{1}(W)}=S_{\sigma} \cap \Lambda_{S}$, so

$$
\int_{Z_{6}}[W]=\int_{S} \operatorname{deg}\left(\left.\left(p \circ \phi_{1}\right)\right|_{W}\right)\left[S_{\sigma} \cap \Lambda_{S}\right],
$$

and the factor $\widetilde{n}(\sigma)$ is $\operatorname{deg}\left(\left.\left(p \circ \phi_{1}\right)\right|_{W}\right)=\operatorname{deg}\left(\left.\phi_{1}\right|_{W}\right) \cdot \operatorname{deg}\left(\left.p\right|_{\phi_{1}(W)}\right)$.

When $p: Z \rightarrow S$ is curvilinear, there is a natural closed inclusion $Z_{k} \subset Z^{\times k}=$ $Z \times{ }_{S} \cdots \times_{S} Z$, which is an isomorphism outside the diagonals. Each permutation between the factors of $Z^{\times k}$ induces an automorphism of $Z_{k}$ (see 4.7.2. of [18]). In our case, when $\sigma=\left(k_{1}, \ldots, k_{r}\right)$ and $r \geq 3$, then $\left.p\right|_{\phi_{1}(W)}$ is curvilinear; $W$ is included in the intersection

$$
\Delta_{\sigma}=\Delta_{1, \ldots, k_{1}} \cap \Delta_{k_{1}+1, \ldots, k_{1}+k_{2}} \cap \cdots \cap \Delta_{k_{1}+\ldots+k_{r-1}+1, \ldots, 6} \subset Z^{\times 6}
$$

(see 3.3 of [7]) and, this time, the automorphisms of $W$ are the permutations interchanging diagonals of the same size.

Since the image by $\phi_{1}$ of a 6 -tuple in $W$ is its first element, then:

- $\operatorname{deg}\left(\left.\phi_{1}\right|_{W}\right)$ is the number of automorphisms of $W$ fixing the first coordinate (see 3.3 of [7]),

- if the first element on the partition $\sigma=\left(k_{1}, k_{2}, \ldots, k_{r}\right)$ is repeated $l$ times, then $\operatorname{deg}\left(\left.p\right|_{\phi_{1}(W)}\right)=l$.

Lemma 5.5. With the previous notation,

$$
\int_{Z_{6}}\left[X_{6} \cap V_{\sigma} \cap \Pi\right]=\int_{Z_{6}}\left[X_{6}\right] \cdot\left[V_{\sigma}\right] \cdot[\Pi] .
$$

Proof. The cycle $\left[X_{6} \cap V_{\sigma} \cap \Pi\right]$ is zero-dimensional (we have seen that

$$
\int_{Z_{6}}\left[X_{6} \cap V_{\sigma} \cap \Pi\right]=\int_{S} \tilde{n}(\sigma) \cdot\left[S_{\sigma} \cap \Lambda_{S}\right]
$$

and that $\left[S_{\sigma} \cap \Lambda_{S}\right]$ is zero-dimensional). We recall from 8.1.11 of [9] that if $U$ and $V$ are two non-singular varieties, it is enough that the intersection is transversal at generic points in order to have $[U \cap V]=[U] \cdot[V]$. In our case, this result applies because:

- $\Pi=\phi^{-1}(\Lambda)$ is the inverse image by the smooth morphism $\phi$ of a linear space $\Lambda \subset \mathbf{P}^{19}$ and therefore $\Pi$ intersects every component of $X_{6} \cap V_{\sigma}$.

- The full intersection is tranversal at each point, because their image by $\phi$ in $\mathbf{P}^{19}$ is tranversal.

- $X_{6} \cap V_{\sigma}$ is also tranversal since, if it were not, $X_{6} \cap V_{\sigma} \cap \Pi$ and its image in $\mathbf{P}^{19}$ will be non-reduced and this is not the case. 
Now, the proof of Theorem 5.1 is complete and the problem of getting the degree of a stratum $C_{\sigma}$ consists in determining $\left[X_{6}\right]$ and computing $\left[X_{6}\right] \cdot\left[V_{\sigma}\right] \cdot[\Pi]$ in the ring $A^{*}\left(Z_{6}\right)$. We are going to get $\left[X_{6}\right]$ using a result by Z . Ran [24] based on the formula of residual intersection of W. Fulton and D. Laksov [10].

Proposition 5.6. With the previous notation,

$$
\left[X_{6}\right]=\Pi_{j=1}^{6}\left(\left(p_{j, j}^{j}\right)^{*}([X])-\left[D^{j, 6}\right]\left\{\frac{\left(p_{j, j}^{j}\right)^{*} c(E)}{1+\left[D^{j, 6}\right]}\right\}_{1}\right)
$$

where $p_{j, j}^{j}=p_{2,2}^{2} \circ p_{2,3}^{3} \circ \cdots \circ p_{2, j}^{j}, D^{j, 6}=D_{1, j}^{6}+D_{2, j}^{6}+\cdots+D_{j-1, j}^{6}$ and $D_{i, j}^{6}=$ $\left(p_{i, j}^{6}\right)^{*} D_{1, j-i+1}^{j-i+1}$ with $p_{i, j}^{6}$ a composition of projections from $Z_{6}$ to $Z_{j-i+1}$ onto the first factor if $i=1$ and onto the second factor if $i>1$.

Proof. To get $\left[X_{6}\right]$ we can apply Theorem (4.2) from 24 because:

- since $X=\left(s_{E}\right)_{0}$ where $s_{E}$ is a section of the vector bundle $E$ over $Z$, then $c\left(N_{X} Z\right)=j^{*} c(E)$.

- the morphisms $X_{t} \rightarrow X_{t-1}$ induced by $f: X \rightarrow S$ are local complete intersection (l.c.i.) of the same codimension, for $t=1, \ldots, k$, so the residual intersection formula of [10] applies. To see this, we first note that $f$ is l.c.i. of codimension 0 because $X \hookrightarrow Z$ is a closed regular inclusion of codimension 2 and $Z$ is smooth over $S$ with relative dimension 2. Now, in general, if $X$ is Cohen-Macaulay and $f$ is l.c.i. of codimension $n$, then a necessary and sufficient condition for every morphism $X_{t} \rightarrow X_{t-1}$ with $t=1, \ldots, k$, to be l.c.i. of codimension $n$ is that all of these morphisms are of the same codimension as $f$ (see [17]). As we saw in $\S 3$, $X$ is smooth and $X$ is also the zero scheme of a regular section $s_{E}$ of the vector bundle $E$ over $Z$. In this case, by the inductive constructions of $\$$, each $X_{t}$ is again smooth and it is also the zero locus of a regular section of the secant bundle $E_{t}$ over $Z_{t}$, so $\operatorname{codim}\left(X_{t}, Z_{t}\right)=\operatorname{rank}\left(E_{t}\right)$ (for $Z_{t}$ is Cohen-Macaulay (in fact, smooth)) and, finally $X_{t} \rightarrow X_{t-1}$ is l.c.i. of codimension 0 for all $t$. The formula of residual intersection $((4.2)$ of $[24])$ says that

$$
\left[X_{k}\right]=\Pi_{j=1}^{k}\left(\left(p_{j, j}^{j}\right)^{*}([X])-\left[D^{j, k}\right]\left\{\frac{\left(p_{j, j}^{j}\right)^{*} c(E)}{1+\left[D^{j, k}\right]}\right\}_{2-1}\right),
$$

where $\{\cdot\}_{r}$ denotes the homogeneous component of $\operatorname{dim} r$ and $c(E)$ and $[X]=c_{2}(E)$ are known (see Lemma 3.10 ).

Lemma 5.7. If $V_{\sigma}$ is the intersection of (the pullback of) the exceptional divisors $E_{1}, \ldots, E_{j}$, then $\left[V_{\sigma}\right]=\left[E_{1}\right] \cdots\left[E_{j}\right]$.

Proof. Using 8.1.11 of [9], as in Lemma [5.5] it is enough to note that the intersection of exceptional divisors $V_{\sigma}=\bigcap_{l=1}^{j} E_{l}$ is not only transversal but it is smooth.

\section{Effective computation of Degree And INTERSECTION RING OF SPACES FOR $k$-TUPLES}

In order to determine $\operatorname{deg}\left(C_{\sigma}\right)$ we have to compute $\left[X_{6}\right] \cdot\left[V_{\sigma}\right] \cdot[\Pi]$ in $A^{*}\left(Z_{6}\right)$.

In general, if $Z$ is a projective bundle over $S$ and $A^{*}(S)$ is known, we can determine completely $A^{*}\left(Z_{k}\right)$ for any $k=1,2, \ldots$ (see Proposition 6.2) and, in our case, it turns out to be a polynomial ring on 23 variables, modulo 38 relations, so effective computations in this ring, using Gröbner bases' methods are very costly. 
In spite of this fact, in order to effectively compute the product $\left[X_{6}\right] \cdot\left[V_{\sigma}\right] \cdot[\Pi]$ in $A^{*}\left(Z_{6}\right)$ it is not necessary to know the whole ring, as we will see in a moment.

In this section the notation is the same as in 4 , writing $e c_{k, 1}:=\left[D_{1, k}^{k}\right]$ for the class of the exceptional divisor in $Z_{k}$. We will apply several times a result by S. Keel [15] about the intersection ring of a blown-up variety, saying that, if $i: X \hookrightarrow Y$ is a regular inclusion of codimension $d, i^{*}: A^{*}(Y) \rightarrow A^{*}(X)$ is surjective and $\widetilde{Y} \rightarrow Y$ is the blow up of $Y$ along $X$ with exceptional divisor $\widetilde{X}$, then

$$
A^{*}(\widetilde{Y}) \cong \frac{A^{*}(Y)[T]}{\left(P(T), T \cdot \operatorname{ker}\left(i^{*}\right)\right)}
$$

where $P(T) \in A^{*}(Y)[T]$ is any polynomial with constant term equal to $[X]$ and whose restriction to $A^{*}(X)$ is the Chern polynomial of the normal bundle $N_{X} Y$.

Proposition 6.1. Let $p: Z \rightarrow S$ be a smooth morphism and suppose that $A^{*}(Z)$ is a finitely generated $A^{*}(S)$-module. For $k=1,2, \ldots$ let $a \in A^{*}\left(Z_{k}\right)$ be a zerodimensional cycle consisting in a product of pullbacks of cycles from $A^{*}\left(Z_{j}\right)$ with $j \leq k$ and some power of the class of the exceptional divisor in $Z_{k}$. Then, the degree of the cycle a can be computed as the degree of a zero-dimensional cycle in $A^{*}(Z)$.

Proof. We prove this by induction in $k$. The cases $k=1,2$ are easy to work out, so let's suppose that we can determine the degree of a zero-dimensional cycle in $A^{*}\left(Z_{k}\right)$ going down to $A^{*}\left(Z_{k-1}\right)$ and let's prove that we can do the same with one in $A^{*}\left(Z_{k+1}\right)$. By Keel's result (6.1) described before, $a=e c_{k+1,1}^{\alpha} \cdot b_{k+1}^{*} c$ with $c \in A^{*}\left(Z_{k} \times_{Z_{k-1}} Z_{k}\right)$. We distinguish two cases:

1. $\alpha=0$. In this case, as $b_{k+1 *} b_{k+1}^{*} c=c$ (see 6.7.(b) of [9]), we have

$$
\int_{Z_{k+1}} a=\int_{Z_{k+1}} b_{k+1}^{*} c=\int_{Z_{k} \times Z_{k-1} Z_{k}} c=\int_{Z_{k}} p r_{1 *} c .
$$

To get $p r_{1 *} c$, we recall the diagram

$$
\begin{array}{lllll}
E_{k+1} & \stackrel{j_{k+1}}{\longrightarrow} & Z_{k+1} & & \\
g_{k+1} \downarrow & & \downarrow^{b_{k+1}} & & \\
\Delta_{k} & \stackrel{l_{k}}{\longrightarrow} & Z_{k} \times Z_{k-1} Z_{k} & \stackrel{p r_{2}}{\longrightarrow} & Z_{k} \\
& & \downarrow^{p r_{1}} & & \downarrow^{\iota_{k-1} \circ p_{1, k-1}^{k}} \\
& & Z_{k} & \stackrel{p_{2, k}^{k}}{\longrightarrow} & Z_{k-1}
\end{array}
$$

Since, by hypothesis $c \in A^{*}\left(Z_{k} \times Z_{k-1} Z_{k}\right)$ can be written as $c=p r_{1}^{*} d \cdot p r_{2}^{*} e$ with $d, e \in A^{*}\left(Z_{k}\right)$ then, denoting by $q_{1}:=\iota_{k-1} \circ p_{1, k-1}^{k}$ and $q_{2}:=p_{2, k}^{k}$ and using the projection formula, we have

$$
\int_{Z_{k}} p r_{1 *} c=\int_{Z_{k}} d \cdot p r_{1 *} p r_{2}^{*} e=\int_{Z_{k}} d \cdot q_{2}^{*} q_{1 *} e,
$$

where the last equality is (1.7) of 9 .

To know the product $d \cdot q_{2}^{*} q_{1 *} e \in A^{*}\left(Z_{k}\right)$ we need to determine $q_{1 *} e$. Now, by Keel's result (6.1) again, $e \in A^{*}\left(Z_{k}\right)$ must be $e=e c_{k, 1}^{\gamma} \cdot b_{k}^{*} u$ for some $\gamma$, with $u \in A^{*}\left(Z_{k-1} \times_{Z_{k-2}} Z_{k-1}\right)$. Then:

(a) If $\gamma>0, q_{1 *} e=\iota_{k-1 *} \circ p r_{1 *} \circ b_{k *}\left(e c_{k, 1}^{\gamma} \cdot b_{k}^{*} u\right)=\iota_{k-1 *}\left(p r_{1 *}\left(u \cdot b_{k *}\left(e c_{k, 1}^{\gamma}\right)\right)\right)$ by projection formula. Writing $U:=p r_{1 *}\left(u \cdot b_{k *}\left(e c_{k, 1}^{\gamma}\right)\right)$, we have that $U=(-1)^{\gamma-1} m_{\gamma-2} \cdot l_{k-1}^{*} u \in A^{*}\left(Z_{k-1}\right) \cong A^{*}\left(\Delta_{k-1}\right)$, being $m_{\gamma-2}$ the term of degree $\gamma-2$ en $c\left(N_{\Delta_{k-1}}\right)^{-1}$ (see 4.2.2. of [9]). 
It is easy to get $\bar{U}$ such that $U=\iota_{k-1}^{*} \bar{U}$, and then: $q_{1 *} e=\bar{U}$ so

$$
\int_{Z_{k+1}} a=\int_{Z_{k} \times Z_{k-1} Z_{k}} c=\int_{Z_{k}} d \cdot q_{2}^{*} \bar{U}
$$

which is known by induction.

(b) If $\gamma=0, q_{1 *} e=\iota_{k-1 *} \circ p r_{1 *} \circ b_{k *} b_{k}^{*} u=\iota_{k-1 *} \circ p r_{1 *} u$. Since $u \in$ $A^{*}\left(Z_{k-1} \times_{Z_{k-2}} Z_{k-1}\right)$, we have again, by hypothesis, that $u=p r_{1}^{*} f \cdot p r_{2}^{*} h$ with $f, h \in A^{*}\left(Z_{k-1}\right)$ and, consequently $p r_{1 *} u=f \cdot p r_{1 *} p r_{2}^{*} h$. Calling now $V:=p r_{1 *} u$, and working as in (a), we find

$$
\int_{Z_{k+1}} a=\int_{Z_{k} \times Z_{k-1} Z_{k}} c=\int_{Z_{k}} d \cdot q_{2}^{*} \bar{V}
$$

which is known again by induction.

2. If $a=e c_{k+1,1}^{\alpha} \cdot b_{k+1}^{*} c$ with $\alpha>0$, then the situation is similar to (a) when we computed $U$, so using 4.2.2. of [9], we conclude

$$
\int_{Z_{k+1}} a=\int_{Z_{k}} p r_{1 *} \circ b_{k+1 *} a=\int_{Z_{k}}(-1)^{\alpha-1} m_{\alpha-2} \cdot l_{k}^{*} c
$$

(where again $m_{\alpha-2}$ is the term of degree $\alpha-2$ in $c\left(N_{\Delta_{k}}\right)^{-1}$ ), and this is known by induction.

In order to effectively apply Proposition 6.1 we need to determine $c\left(N_{\Delta_{k}}\right)$ for every $k$. From $\$ 4$, we know that $N_{\Delta_{k}}$ is isomorphic to the relative tangent bundle $T_{k}:=T_{p_{2, k}^{k}}$. The total Chern class of $T_{k}$ can be computed inductively, starting with $c\left(T_{1}\right)$ the class of a relative tangent bundle (given by B.5.8. of [9]). Using standard results about Chern classes (see 15.4.(iv), 15.4.2. of [9]), one gets

$$
c\left(T_{k}\right)=\left(1+e c_{k, 1}\right) \sum_{i=0}^{2}\left(1-e c_{k, 1}\right)^{i}\left(p_{1, k-1}^{k}\right)^{*} c_{2-i}\left(T_{k-1}\right) .
$$

Implementing the inductive method given in Proposition 6.1 in Maple [20] we finally get the degree of the strata of singular cubic surfaces with a conic node mentioned in Theorem 5.1

\begin{tabular}{|c|c|c|c|c|c|c|c|}
\hline$\sigma$ & $1^{6}$ & $21^{4}$ & $31^{3}$ & $2^{2} 1^{2}$ & $41^{2}$ & 321 & $2^{3}$ \\
\hline Singularity & $A_{1}$ & $2 A_{1}$ & $A_{1} A_{2}$ & $3 A_{1}$ & $A_{1} A_{3}$ & $2 A_{1} A_{2}$ & $4 A_{1}$ \\
\hline Parameters & 3 & 2 & 1 & 1 & 0 & 0 & 0 \\
\hline $\operatorname{deg}\left(C_{\sigma}\right)$ & 32 & 280 & 1200 & 800 & 2200 & 3420 & 305 \\
\hline
\end{tabular}

Note that for $\sigma=(2,2,2)$, we have substracted the contribution of surfaces with a uninode which is $\frac{1}{4} \operatorname{deg}\left(C_{U}\right)$, according to Theorem 5.1 The degree of the stratum $C_{U}, 260$, can be computed separately at least in two ways. First, as a particular case of some formulas for the degree of varieties parametrizing quadrics with vertex of specified dimension, given by I. Vainsencher [28] (1979). Secondly, as we do in 30], using a result by J. Harris and L. W. Tu [11 (1984) about the cohomology class of the degeneracy locus of a symmetric bundle map.

For completeness, we describe here the intersection ring of the space $Z_{k}$ of $k$ tuples of $Z$ with the same image in $S$ (defined in projectivization of a vector bundle $E$ over a smooth scheme $S$. 
Proposition 6.2. Let $Z$ be a projective bundle over a smooth scheme $S$ and let $Z_{k}$ be the space of $k$-tuples of $Z$ over $S$ defined in 8 . Then

$$
A^{*}\left(Z_{k}\right) \cong \frac{A^{*}(S)\left[g_{1}, \ldots, g_{m}\right]}{\left(r_{1}, \ldots, r_{n}\right)}
$$

where $m=\sum_{j=1}^{k} j, n=\sum_{j=1}^{k}(2 j-1) ; k$ of the generators $g_{j}$ are (pullback of) the hyperplane class of $Z$; the rest are (pullback of) exceptional divisors' classes and the relations $r_{j}$ are determined by 3.3.(b), Ex. 8.3.4. of [9] and Keel's result (6.1) above.

Proof. We see this by induction on $k$, using repeatedly 3.3.(b), Ex. 8.3.4. of [9], to determine the ring of a projective bundle and Keel's work (6.1) from [15, for the ring of a blown-up scheme. One starts with the projective bundle $Z$ and its Chow ring $A^{*}(Z)$ written in terms of $A^{*}(S)$. For $k=2$, by construction, the fiber product $Z \times{ }_{S} Z$ is another projective bundle, one finds that $A^{*}\left(Z \times{ }_{S} Z\right) \cong$ $A^{*}(Z) \otimes_{A^{*}(S)} A^{*}(Z)$, and $Z_{2}=B l_{\Delta}\left(Z \times{ }_{S} Z\right)$ is a blown-up projective bundle. When $k=3$ checking that $Z_{2} \times{ }_{Z} Z_{2}$ is a projective bundle over $Z_{2}$ (by 3.4. of [13]) one has that $Z_{3}$ is a projective bundle blown-up two times.

At the step $(k-1)$, one supposes that $Z_{k-1}$ is a projective bundle over $Z_{k-2}$ blown-up $(k-1)$ times and therefore its Chow ring can be written as it is said in the proposition. Now, again by definition, the fiber product $Z_{k-1} \times_{Z_{k-2}} Z_{k-1}$ is another projective bundle over $Z_{k-1}$ blown-up $(k-1)$ times and, finally, for $Z_{k}$ one adds another blow-up getting $A^{*}\left(Z_{k}\right)$ as the ring described in the proposition (for details see [30]).

\section{REFERENCES}

1. A. B. Altman and S. L. Kleiman, Foundations of the theory of Fano schemes, Compos. Math. 34 (1977), 3-47. MR 58:27967

2. V. I. Arnold, Normal forms of functions near degenerate critical points, the Weyl groups $A_{k}$, $D_{k}$ and $E_{k}$ and Lagrangian singularities, Func. Anal. Appl. 6 (1972), 254-272. MR 50:8595

3. V. I. Arnold, Normal forms of functions in the neighborhood of degenerate critical points, Russ. Math. Surv. 29 (2) (1974), 11-49. MR 58:24324

4. J. W. Bruce, A stratification of the space of cubic surfaces, Math. Proc. Cambridge 87 (1980), 427-441. MR 81c: 14022

5. J. W. Bruce and C. T. C. Wall, On the clasification of cubic surfaces, J. Lond. Math. Soc. (2) 19 (1979), 245-256. MR 80f:14021

6. A. Cayley, A memoir on cubic surfaces, Phil. Trans. Roy. Soc. 159 (1869), 231-236.

7. S. J. Colley, Enumerating stationary multiple-points, Adv. Math. 66 (1987), 149-170. MR 89g:14042

8. D. F. Coray and I. Vainsencher, Enumerative formulae for ruled cubic surfaces and rational quintic curves, Comment. Math. Helv. 61 (1986), 510-518. MR 87m:14061

9. W. Fulton, Intersection Theory, Springer-Verlag, (1984). MR 85k:14004

10. W. Fulton and D. Laksov, Residual intersections and the double point formula, Real and complex singularities, ed. P. Holm, Sijthoff \& Nordhoff, (1977), 171-177. MR 58:27971

11. J. Harris and L. W. Tu, On symmetric and skew-symmetric determinantal varieties, Topology, Vol. 23, No.1 (1984), 71-84. MR 85c: 14032

12. R. Hartshorne, Algebraic Geometry, Springer-Verlag, (1977). MR 57:3116

13. S. Iitaka, Algebraic Geometry, Springer Verlag, (1982). MR 84j:14001

14. S. Katz and S. A. Stromme, Schubert: a MAPle package for intersection theory. Available by anonymous ftp from ftp.math.okstate.edu or linus.mi.uib.no, cd pub/schubert.

15. S. Keel, Intersection theory of moduli space of stable $N$-pointed curves of genus cero, Trans. Amer. Math. Soc. 330 (1992), 545-574. MR 92f:14003

16. S. L. Kleiman, The enumerative theory of singularities, Real and complex singularities, ed. P. Holm, Sijthoff \& Nordhoff, (1977), 297-396. MR 58:27960 
17. S. L. Kleiman, Multiple-point formulas. I: Iteration, Acta Math. 147 (1981), 13-49. MR 83j:14006

18. S. L. Kleiman, Multiple-point formulas. II: the Hilbert scheme, Enumerative Geometry, (Proc. Conf., Sitges, 1987), ed. S. Xambó Descamps, LNM 1436, Springer-Verlag (1989), 101-138. MR 92a:14062

19. D. Laksov, Secant bundles and Todd's formula for the double points of maps into $\mathbf{P}^{n}$, Proc. Lond. Math. Soc. 37 (1978), 120-142. MR 80c:14007

20. MAPLE V, Copyright (c), 1981-1990, University of Waterloo.

21. A. Mattuck, Secant bundles on symmetric products, Am. J. Math. 87 (1965), 779-797. MR 33:7345

22. J. M. Miret and S. Xambó Descamps, On the geometry of nodal plane cubics: the condition $p$, Enumerative Geometry: Zeuthen Symposium, ed. S. L. Kleiman y A. Thorup, Contemp. Math. 123, A.M.S. (1991), 169-187. MR 93e:14064

23. J. M. Miret and S. Xambó Descamps, Rational equivalence on some families of plane curves, Ann. I. Fourier, Grenoble, 44, 2 (1994), 323-345. MR 95g:14006

24. Z. Ran, Curvilinear enumerative geometry, Acta Math. 155 (1985), 81-101. MR 86m:14040

25. L. G. Roberts, Lines in singular cubics, Queen's Papers in Pure and Appl. Math.

26. L. Schläfli, On the distribution of surfaces of the third order into species, Phil. Trans. Roy. Soc. 153 (1864), 193-247.

27. R. L. E. Schwarzenberger, The secant bundle of a projective variety, Proc. London. Math. Soc. 14 (1964), 369-384. MR 28:3042

28. I. Vainsencher, The degrees of certain strata of the dual variety, Compos. Math. 38 (1979), 241-252. MR 81g:14024

29. I. Vainsencher, Counting divisors with prescribed singularities, Tran. Amer. Math. Soc., Vol. 267, No. 2, (1981), 399-422. MR 83b:14021

30. María J. Vázquez-Gallo, Grado de variedades de superficies singulares, Ph.D. thesis, University Autonoma of Madrid (1997).

Departamento de Matematicas, Facultad de Ciencias, Universidad Autonoma de MADRID, MADRID, 28049, SPAIN

E-mail address: rafael.hernandez@uam.es

Departamento de Matematicas, Facultad de Ciencias, Universidad Autonoma de MADRID, MADRID, 28049, SpAIN

E-mail address: mjesus.vazquez@uam.es 